\title{
Urinary kidney injury molecule-1 rapid test predicts acute kidney injury in extremely low-birth-weight neonates
}

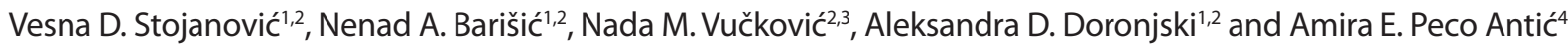

BACKGROUND: The new urinary and serum biomarkers are discovered and are being investigated. With them we can diagnose acute kidney injury (AKI) faster and more precisely and they also have a significant role in the outcome prediction.

METHODS: The study included 22 extremely low-birthweight neonates who were hospitalized in the neonatal intensive care units. They were divided into two groups based on serum creatinine $(\mathrm{SCr})$ level — with and without AKI. Detection and quantification of urinary kidney injury molecule-1 (UKIM-1) was done on the third day of life, using commercially available KIM-1 rapid test. Subsequently, measurements were repeated only in subjects who were diagnosed with AKI, at different values of SCr.

RESULTS: Logistic regression analysis showed that AKI is an independent risk factor for mortality. In a group of neonates with AKI, 50\% of neonates administered the KIM-1 rapid test showed positive findings. KIM-1 rapid test was positive in patients with a wide range of SCr levels (range of 78.73-385 $\mu \mathrm{mol} / \mathrm{l})$, but all subjects had oliguria and died in the next $24 \mathrm{~h}$. CONCLUSION: KIM-1 is a significant predictor of death. On the other hand, our study failed to prove that KIM-1 rapid test has any significance for early prediction of AKI.

A cute kidney injury (AKI) occurs in $2.4-56 \%$ neonates treated in neonatal intensive care units (NICU). In neonates with AKI, mortality rate ranges from $33 \%$ up to $78 \%$ (1-4). AKI is an independent predictor of mortality in critically ill newborns. It is believed that AKI leads not only to disturbances of homeostasis of fluids and electrolytes, but also, by suppressing systemic inflammatory response, plays a key role in the systemic derangement in multiorgan failure $(5,6)$. Infants who survived AKI in neonatal period, often have longterm sequelae that include hypertension, renal tubular acidosis, impared renal growth, and development or chronic renal failure of different degrees $(7,8)$.

The criteria for the diagnosis of AKI are based solely on the basis of serum creatinine levels (7). Serum creatinine (SCr) levels in premature infants cannot be considered as adequate for the diagnosis of AKI due to few reasons: in the first two to $3 \mathrm{~d}$ of life SCr levels are a reflection of mother's SCr, after this period SCr levels fall during next few days or even weeks, depending on the gestational age (GA) (9); SCr levels do not change drastically until $50 \%$ of kidney function is lost; if the glomerular filtration rate is very low, there is a secretion of the SCr (10); SCr levels are influenced by many factors including lean muscle mass, hydration status, GA, etc; in patients on dialysis, $\mathrm{SCr}$ is no longer valid marker for evaluation of renal function, because it is dialyzed as well (11).

In the future, when new biomarkers of AKI (neutrophil gelatinase-associated lipocalin-NGAL, urinary interleukin-18, kidney injury molecule-1 (KIM-1), serum cystatin, etc.) become widely introduced in routine practice, the present definition of AKI will be indisputably expanded and upgraded, that will provide a possibility for early diagnosis of AKI well before $\mathrm{SCr}$ levels rise (6). In the meantime, until early biomarkers of AKI become routinely used, dynamics of $\mathrm{SCr}$ must be closely monitored in order to timely diagnose AKI and assess the severity of the disease (12).

KIM-1 is a type I transmembrane protein and is highly upregulated in the proximal tubule epithelial cells. KIM-1 mRNA and protein are expressed at a low level in normal kidney but are increased in postischemic kidney. KIM-1 is also a tissue and urinary biomarker for nephrotoxicant-induced kidney injury $(13,14)$. These cells undergo regeneration after various forms of injury, and shed KIM-1 antigen into urine. KIM-1 plays a role in epithelial adhesion, growth and differentiation. KIM-1 has a role in the process of regeneration after damage to the epithelium and in the removal of dead cells from the tubular lumen by phagocytosis. Injury of renal tubules leads to increased concentration of urinary KIM-1 $(15,16)$.

To determine whether urinary KIM-1 (uKIM-1) can be used to detect AKI in extremely low-birth-weight (ELBW) neonates, we used uKIM-1 rapid test. We evaluated prognostic value of this biomarker for prediction of AKI and mortality.

\section{RESULTS}

Out of 30 ELBWs that were hospitalized in our NICU during the study period, 8 met the exclusion criteria (prematurely born infants who died in first $72 \mathrm{~h}$ or those with congenital

'Intensive Care Unit, Institute for Child and Youth Health Care of Vojvodina, Novi Sad, Serbia; ${ }^{2}$ Faculty of Medicine University of Novi Sad, Novi Sad, Serbia; ${ }^{3}$ Centre of Pathology and Histology, Clinical Centre of Vojvodina, Vojvodina, Serbia; ${ }^{4}$ Department of Nephrology, University Children Hospital, Faculty of Medicine University of Belgrade, Belgrade, Serbia. Correspondence: Vesna D. Stojanović (vsnefro@gmail.com) 
malformations of the urinary system), so the final study group involved a total of 22 patients, of which 10 (45\%) had AKI.

On average, the diagnosis of AKI was set in 7 th day of life $(7.1 \pm 5.11)$. In 8 out of $10(80 \%)$ neonates, the diagnosis of AKI was set in the first $7 \mathrm{~d}$ of life. The mean value of SCr on the third

Table 1. Characteristics of neonates with and without AKI

\begin{tabular}{lccc}
\hline & AKI $(n=10)$ & Non-AKI $(n=12)$ & $P$ \\
\hline GA (weeks) & $25.88 \pm 1.78$ & $26.39 \pm 1.81$ & 0.424 \\
Birth weight (g) & $798.00 \pm 152.37$ & $824.54 \pm 172.45$ & 0.604 \\
AS first minute & $2.1 \pm 1.1$ & $3.81 \pm 1.1$ & $0.003^{\mathrm{a}}$ \\
AS fifth minute & $4.2 \pm 1.58$ & $6.0 \pm 1.28$ & $0.012^{\mathrm{a}}$ \\
Gender (female/male) & $3 / 7$ & $6 / 6$ & 0.415 \\
\hline
\end{tabular}

aStatistically significant difference.

AKI, acute kidney injury; AS, Apgar scores.

Table 2. Comorbidities in neonates with and without AKI

\begin{tabular}{lccc}
\hline & $\begin{array}{c}\text { AKI } \\
(n=10)\end{array}$ & $\begin{array}{c}\text { Non-AKI } \\
(n=12)\end{array}$ & $P$ \\
\hline Hypernatriemia & $8 / 10(80 \%)$ & $4 / 12(33.3 \%)$ & $0.043^{\mathrm{a}}$ \\
Septicemia & $8 / 10(0 \%)$ & $7 / 12(58.3 \%)$ & 0.381 \\
Oliguria & $5 / 10(50 \%)$ & $0 / 12(0 \%)$ & $0.009^{*}$ \\
Necrotizing enterocolitis & $8 / 10(80 \%)$ & $5 / 12(41.6 \%)$ & 0.099 \\
Intracranial hemorrhage & $10 / 10(100 \%)$ & $4 / 12(33.3 \%)$ & $0.0017^{\mathrm{a}}$ \\
Patent ductus arteriosus & $1 / 10(10 \%)$ & $1 / 12(8.3 \%)$ & 1 \\
\hline aStatistically significant difference. & & & \\
AKI, acute kidney injury. & & \\
*The P values that are statistically significant. & &
\end{tabular}

Table 3. Drugs commonly used in neonates with and without AKI

\begin{tabular}{lccl}
\hline & AKI $(n=10)$ & Non-AKI $(n=12)$ & \multicolumn{1}{c}{$P$} \\
\hline Dopamine & $9 / 10(90 \%)$ & $5 / 12(41.6 \%)$ & $0.031^{\mathrm{a}}$ \\
Furosemide & $10 / 10(100 \%)$ & $1 / 12(8.3 \%)$ & $0.0001^{\mathrm{a}}$ \\
Ceftazidime & $7 / 10(70 \%)$ & $4 / 12(33.3 \%)$ & 0.198 \\
Vacomycine & $2 / 10(20 \%)$ & $0 / 12(0 \%)$ & 0.195 \\
Colystine & $6 / 10(60 \%)$ & $0 / 12(0 \%)$ & $0.0028^{\mathrm{a}}$ \\
\hline
\end{tabular}

aStatistically significant difference.

AKI, acute kidney injury. day of life in a group of patients with AKI was $112.02 \pm 28.07$ and $95.22 \pm 14.64 \mu \mathrm{mol} / \mathrm{l}(P=0.111)$ in the group of patients without AKI. There were no patients who received renal replacement therapy.

The average increase in SCr levels from the first to third day of life, in neonates who were diagnosed with AKI during the first $7 \mathrm{~d}$ of life, was $44.625 \pm 18.438 \mu \mathrm{mol} / \mathrm{l}$, and in neonates without AKI was $22.625 \pm 23.82 \mu \mathrm{mol} / \mathrm{l}(P=0.0498)$. Table 1 shows characteristics of neonates with and without AKI. Neonates with AKI had significantly lower values of Apgar scores (AS) in first (2.1 vs. 3.8; $P=0.03)$ and fifth minute (4.2 vs. $6.0 ; P=0.012)$ compared to infants without AKI. These two groups of infants did not differ in birth weight $(B W)$, gestational age (GA), and gender.

Table 2 shows comorbidities in patients with and without AKI. Neonates with AKI have been more prone to hypernatriemia ( 80 vs. $33.3 \%, P=0.043$ ) and intracranial hemorrhage (100 vs. $33.3 \% ; P=0.0017$ ) compared to infants without AKI. Half (50\%) of the neonates with AKI had oliguria.

All those conditions were diagnosed before the diagnosis of AKI was set. All neonates were intubated and mechanicaly ventilated and received surfactant endotrachealy in the first day of life.

Table 3 shows drugs used in both groups of patients, with and without AKI. In comparation to neonates without AKI, neonates with AKI were more often exposed to dopamine (90 vs. $41.6 \% ; P=0.031$ ), furosemide ( 100 vs. $8.3 \%, P=0.0001$ ) and colomycine ( 60 vs. $0 \% ; P=0.0028$ ) before the diagnosis of AKI was set. None of the neonates, from both groups, received nonsteroidal anti-inflammatory drugs. All neonates were initially treated with ampicillin and gentamycin.

In neonates with $\mathrm{AKI}$, mortality rate was significantly higher compared to infants without AKI (80\% (8/10) vs. $25 \%(3 / 12)$; $P=0.03)$.

Logistic regression analysis showed that AKI is an independent risk factor for mortality (OR 12.00, CI 1.581-91.087).

\section{KIM-1 Rapid Test}

In a group of neonates with AKI $(n=10)$, in five (50\%) neonates KIM-1 rapid test showed positive findings, and in remaining five (50\%) subjects results were negative. In all neonates

Table 4. Serum creatinine levels in neonates who had AKI, with positive and negative KIM-1 rapid test

\begin{tabular}{|c|c|c|c|c|c|c|c|c|c|c|}
\hline & 1.KIM-1+ & 2.KIM-1+ & 3.KIM-1+ & 4.KIM-1+ & 5.KIM-1+ & 1.KIM-1- & 2.KIM-1- & 3.KIM-1- & 4.KIM-1 & 5.KIM-1- \\
\hline $\begin{array}{l}\text { Age at the time of } \\
\text { AKI diagnosis (days) }\end{array}$ & 6 & 8 & 4 & 7 & 4 & 21 & 4 & 6 & 7 & 4 \\
\hline $\begin{array}{l}\text { KIM-1 positive } \\
\text { (age (days) and } \\
\text { sCr levels }(\mu \mathrm{mol} / \mathrm{I}) \text { ) }\end{array}$ & $6 / 173.24$ & $16 / 385$ & $7 / 237$ & $18 / 78.73$ & $7 / 278.2$ & / & / & / & / & / \\
\hline $\begin{array}{l}\text { KIM-1 negative } \\
\text { (age (days) and } \\
\text { sCr levels }(\mu \mathrm{mol} / \mathrm{l}) \text { ) }\end{array}$ & $\begin{array}{l}\text { 3/108.58; } \\
4 / 135.4 \\
5 / 137.86\end{array}$ & $\begin{array}{c}3 / 102.2 ; 8 / 206.3 ; \\
9 / 237.1 ; \\
12 / 297.0 ; 14 / 346\end{array}$ & $\begin{array}{l}\text { 3/150.8; } \\
\text { 4/200.5; } \\
6 / 235\end{array}$ & $\begin{array}{l}3 / 69.04 ; 5 / 89.67 ; \\
7 / 185.34 ; 17 / 45\end{array}$ & $\begin{array}{l}3 / 155.1 \\
5 / 218.7 \\
6 / 26.01\end{array}$ & $\begin{array}{l}3 / 105.2 \\
21 / 132.0 \\
24 / 163.4\end{array}$ & $\begin{array}{l}\text { 3/136.0; } \\
\text { 4/153.5; } \\
6 / 185.3\end{array}$ & $\begin{array}{c}3 / 83.9 \\
7 / 132.8 \\
8 / 144.9\end{array}$ & $\begin{array}{l}3 / 115.9 \\
7 / 183.2 \\
11 / 214.6\end{array}$ & $\begin{array}{l}\text { 3/93.4; } \\
5 / 171 \\
6 / 120.3\end{array}$ \\
\hline $\begin{array}{l}\text { Normalization of } \\
\text { renal function }\end{array}$ & No & No & No & No & No & No & Yes & Yes & Yes & Yes \\
\hline Age of death (days) & 6 & 16 & 7 & 18 & 7 & 24 & 10 & 12 & Survived & Survived \\
\hline
\end{tabular}


Table 5. Mean values of histologic injury scores, in all groups

\begin{tabular}{lccc}
\hline Group & $\begin{array}{c}\text { Proximal } \\
\text { tubules }\end{array}$ & $\begin{array}{c}\text { Distal } \\
\text { tubules }\end{array}$ & Interstitium \\
\hline AKI with positive KIM-1 $(n=3)$ & $2.33(2 ; 3 ; 2)^{\mathrm{a}}$ & $2(1 ; 3 ; 2)^{\mathrm{a}}$ & $1.66(1 ; 3 ; 1)^{\mathrm{b}}$ \\
AKI with negative KIM-1 $(n=3)$ & $1.33(2 ; 1 ; 1)^{\mathrm{a}}$ & $1(1 ; 1 ; 1)^{\mathrm{a}}$ & $0.33(1 ; 0 ; 0)^{\mathrm{b}}$ \\
Without AKI $(n=2)$ & $1.5(1 ; 2)^{\mathrm{a}}$ & $1.5(1 ; 2)^{\mathrm{a}}$ & $0.5(0 ; 1)^{\mathrm{b}}$ \\
\hline
\end{tabular}

aDegree of cellular damage for proximal and distal tubules: 0-no damage; 1-minimal

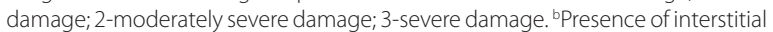
edema: 0-no edema; 1-light edema; 2-moderately prominent edema; 3-marked edema. AKI, acute kidney injury.

that had AKI and positive KIM-1 rapid test, urinary levels of KIM-1 ranged between 250 and $500 \mathrm{pg} / \mathrm{ml}$.

Newborns who had AKI and positive KIM-1 rapid test, more often had oliguria compared to infants who had AKI with negative KIM- 1 rapid test $(5 / 5$ vs. $0 / 5 ; P=0.008)$. The mean values or incidences of other parameters were not statisticaly significantly different between the two groups: GA $(25.96 \mathrm{GW}$ vs. $25.79 \mathrm{GW} ; P=0.891$ ), birth weight ( 808 vs. $788 \mathrm{~g} ; P=0.849$ ), first minute AS ( 2.4 vs. $1.8 ; P=0.421)$, fifth minute AS (4.2 vs. $4.2 ; P=1.0$ ), gender structure (female/male; $1 / 4$ vs. $2 / 3$; $P=1.0$ ), incidence of hypernatremia ( $5 / 5$ vs. $3 / 5 ; P=0.444)$, sepsis ( $5 / 5$ vs. $5 / 3 ; P=0.444)$, nectotizing enterocloitis ( $4 / 5$ vs. $4 / 5, P=1.0)$, intracranial hemorhage ( $5 / 5$ vs. $5 / 5 ; P=1.0)$ and patent ductus arteriousus persistens $(0 / 5$ vs. $1 / 5, P=1.0)$, as also as exposure to drugs such as dopamine (5/5 vs. $4 / 5 ; P=$ 1.0 ), furosemide ( $5 / 5$ vs. $5 / 5 ; P=1.0)$, ceftazidime ( $4 / 5$ vs. $4 / 5$; $P=1.0$ ), vancomycin ( $1 / 5$ vs. $1 / 5 ; P=1.0)$, or colystine ( $4 / 5$ vs. $2 / 5 ; P=0.524)$.

Table 4 shows the age (days of life) of patients at the time they were diagnosed with AKI, age (days of life) and SCr levels when KIM-1 rapid test have been done, and the final outcome.

In all infants who did not have AKI, results of KIM-1 rapid test on the third day of life were negative. In those patients, $\mathrm{SCr}$ values ranged from 60.9 to $110.1 \mu \mathrm{mol} / \mathrm{l}$.

Mortality was similar between the groups of newborns with AKI, with positive and negative KIM- 1 rapid test (5/5 vs. $3 / 5$; $P=0.444)$.

In the group of neonates with AKI and negative KIM-1 rapid test, one neonate died within $24 \mathrm{~h}$ after the diagnosis of AKI was set; four children normalize renal function (but, later in the course of the disease, two infants died due to sepsis with multiorgan dysfunction).

All neonates with AKI and positive KIM-1 rapid test, died within the next $24 \mathrm{~h}$ after KIM- 1 became positive.

\section{Histopahological Findings}

Mean values of histologic injury scores, in all groups, are shown in Table 5.

\section{DISCUSSION}

Critically ill neonates are at great risk to develop AKI because they are often exposed to a number of nephrotoxic drugs and often have infections that lead to multiorgan dysfunction $(17,18)$. In critically ill neonates, AKI usually occurs in the first week of life (80.5\%) (12), which was also confirmed in our study (80\%).

Neonatal AKI is a serious problem in critically ill neonates and is associated with poor outcome. The mortality is very high, particularly poor prognosis and high mortality rates have neonates with oliguric AKI (19). In our study, all neonates that had oliguria died.

It was also found that AKI is a predictor of mortality in ELBW neonates. In their study, Askenazi et al. (20) found that any increase in SCr to $88.4 \mu \mathrm{mol} / \mathrm{l}$ was associated with a twofold increase of odds of death. In one prospective study conducted by Mathur et al. (21), it was found that the incidence of AKI among neonates with sepsis was $26 \%$ and that the mortality rate was higher among neonates with AKI compared to those without AKI. Koralkar et al. (12) also confirmed that mortality is significantly higher in neonates with AKI.

Few studies have examined risk factors for AKI in ELBW neonates. In the study of Askenazi et al. (20), very-low-birthweight neonates with and without AKI did not differ in BW, GA, gender, Apgar score in first and fifth minute or exposure to nephrotoxic drugs (vancomycin, aminoglicozides, and indomethacin), but neonates with AKI had significantly lower serum sodium levels and higher incidence of intraventricular hemorrhage. In our study, the ELBW neonates with AKI had significantly lower values of AS in the first and in the fifth minute, significantly higher incidence of intracranial hemorrhage and significantly more often were treated with inotrope (dopamine), furosemide, and colystine. Colystine is nephrotoxic, and the majority of newborns with AKI had to receive it due to sepsis caused by Acineobacter spp. Due to the presence of contraindications, there were no patients, in neither group, that received nonsteroidal anti-inflammatory drugs (ibuprofen). In other studies, a significantly higher number of neonates with AKI previously received ibuprofen, and therapy with ibuprofen was stressed out as a risk factor for the development of AKI (1).

Aggarwal et al. (22) also found that the incidence of AKI in neonates with AS $\leq 6$ in the fifth minute is significantly higher than in controls. In a group of neonates with AS $\leq 6$, Gupta et al. (19) found that the incidence of AKI was $47 \%$ and mortality was $14.1 \%$. Those findings were confirmed by similar results of other authors $(1,23,24)$. Choker et al. (25) found that neonates with AKI had significantly lower GS and BW than those without AKI.

In our study, infants with AKI more often had hypernatriemia prior diagnosis of AKI. In a study of Stojanović et al. (26), it was proven that newborns with AKI have significantly higher serum sodium levels in the third and fourth day of life compared to infants without AKI. Low fluid intake, and especially increased insensibile fluid loss in ELBW neonates may rapidly lead to dehydration (and consequently, high serum sodium levels), which is the major cause of neonatal AKI $(7,27)$.

Diagnostic criteria of AKI are still based on the increase in the SCr and/or urine output $(12,20)$. Unfortunately, it is well known that the SCr is late biomarker for AKI. In our study, it was found that the increase in the SCr in the first $3 \mathrm{~d}$ of life was 
significantly higher in ELBW neonates who developed AKI in the first week of life. In the study of Choker et al. (25), that encompassed 34 very preterm infants, AKI was defined as an increase in daily SCr levels above $43 \mu \mathrm{mol} / \mathrm{l}$ on day 1 , and/or 21 $\mu \mathrm{mol} / \mathrm{l}$ on day 2 , and/or $14 \mu \mathrm{mol} / \mathrm{l}$ on day 3 , and/or $22 \mu \mathrm{mol} / \mathrm{l}$ on day 4. Larger studies are needed to prove the importance of these data.

The majority of recently published studies have demonstrated that urinary biomarkers (NGAL, KIM-1, and others) can detect infants who are in high-risk group for development of AKI, and that those biomarkers are accurate for prediction of outcome, such as mortality $(6,27)$. The ideal biomarker would be the one that would diagnose AKI immediately after the exposure to etiological factors that may lead to kidney damage and one that is independent of the glomerular filtration rate $(28,29)$.

Several studies have shown that the concentration of certain urinary biomarkers depends on gestational age and birth weight. Recent studies have shown that immature neonates have higher concentrations of these biomarkers in the urine. This is probably due to the inability of the immature tubules to reabsorb proteins $(6,30,31)$. There are very few studies that have examined these biomarkers in the neonates.

Clinical studies revealed that KIM-1 is highly effective at distinquishing true acute tubular necrosis from other types of renal injury $(6,30)$. van Timmeren et al. (32) found induction of tubular KIM-1 expression in various renal diseases, and showed that KIM-1 expression is associated with tubulointerstitial damage, inflammation, and dedifferentiated tubules (which suggest a role for KIM-1 in tubular fibrosis).

Hystopathological kidney analysis was done in eight patients who have died. More severe damage of proximal and distal tubules was detected in patients with AKI and positive KIM1 , and they also had more prominent interstitial edema compared with patients with AKI and negative KIM-1 and patients without AKI.

Most studies indicate that KIM-1 is not as accurate for early prediction of AKI, but it is better for identifying and confirmation of already developed AKI $(6,28)$. Two large studies have shown that KIM-1 is an effective new urinary biomarker for the diagnosis of AKI in the first $24 \mathrm{~h}$ after kidney injury, especially for the diagnosis of ischemic AKI $(15,16)$.

Askenazi et al. (6) examined the different urinary biomarkers as predictors of AKI and mortality in very-low-birthweight infants. Mean values of KIM-1 in neonates with AKI were $867 \mathrm{pg} / \mathrm{ml}$, and without AKI were $835 \mathrm{pg} / \mathrm{ml}$. They have not proven the importance of KIM-1 in the prediction of AKI, but they demonstrated that KIM-1 has the potency to predict mortality in preterm neonates hospitalized in NICU (6).

In another study, Askenazi et al. (6) presented the reference values for KIM-1 in relation to gestational age. For newborn babies, of $26.1-28 \mathrm{wk}$ reference value is $158(117-212) \mathrm{pg} / \mathrm{ml}$, and for neonates born before 26 gestational weeks 226 (184277) $\mathrm{pg} / \mathrm{ml}$ (31). In our study, we used rapid test to quantify urinary KIM-1 concentration (33). In all patients who had AKI and positive KIM-1, urinary KIM-1 levels were somewhere between
$250-500 \mathrm{pg} / \mathrm{ml}$. It is interesting that the KIM-1 rapid test was positive in patients with a wide range of SCr levels (range of 78.73-385 $\mu \mathrm{mol} / \mathrm{l})$, but all subjects that were positive for KIM-1 and had AKI also had oliguria and died in the next $24 \mathrm{~h}$. This confirms the hypothesis that the KIM-1 in a significant predictor of death (and probably true predictor of acute tubular necrosis). On the other hand, our study failed to prove that KIM-1 rapid test has any significance for early prediction of AKI.

Huang et al. (16) found that the levels of the UKIM-1 are the highest in patients with acute ischemic tubular necrosis. Detected values of uKIM-1 among patients vary widely, and are influenced by the time. In their study, Han et al. (15) found that the sensitivity of uKIM- 1 is only $50 \%$ immediately after cardiopulmonary bypass. Two other studies have found much greater sensitivity, of more than $90 \%$, when KIM- 1 is measured 2 and $6 \mathrm{~h}$ after cardiopulmonary bypass. However, it's sensitivity is decreased to $74 \%$ after $12 \mathrm{~h}$ cardiopulmonary bypass $(34,35)$. Larger studies are needed to determine when is the optimal timing for determination and measurement of KIM-1 levels in relation to the exposure to etiological factors that may cause AKI.

Using an animal model of perinatal asphyxia, Stojanovic et al. (36) found that the kidney cystatin $\mathrm{C}$ expression was the most intensive $6 \mathrm{~h}$ after hypoxic insult and declined over the time, and that the expression of KIM-1 was less intensive, reaching the peak value $48 \mathrm{~h}$ after hypoxic event.

In their study, which examined the serum and urinary biomarkers in a group of asphyctic newborns, Sarafidis et al. (30), failed to document any significance of uKIM-1 for the prediction of AKI following asphyxia.

To our best knowledge, this is the first study that has examined whether UKIM-1 rapid test may predict AKI in extremely low birth weight neonates. In our study, the positive uKIM-1 rapid test has been associated with severe AKI (already developed AKI) and mortality. The number of ELBW neonates included in the study was small, so larger studies are needed to evaluate KIM-1 against different clinical endpoints (such as the need for dialysis, mortality, length of hospital stay, progression to chronic renal failure).

Also, it would be very important to have a tool to differentiate prerenal from renal kidney failure, to predict the course of AKI and to follow the effects of treatment. Most of the investigators in this field, believe that only a combination of biomarkers will have potential to define the cause of AKI and provide enough information for early diagnosis of AKI.

Present knowledge about the role and significance of early urinary biomarkers of kidney injury like NGAL, KIM-1, osteopontin, cystatin C, interleukin- 18 , and $\beta-2$ microglobulin is limited. Askenazi et al. (6) found that values of osteopontin, cystatin $C$, and $\beta-2$ microglobulin are significantly higher in neonates with AKI, and that higher concentrations of KIM-1 and osteopontin may be related with higher mortality risk. Further trials, involving larger samples of patients, are needed to differentiate which combination of biomarkers of kidney injury has the highest power to predict early development of AKI. 


\section{METHODS}

The study was designed as prospective study and included 30 prematurely born newborns, with body weight (BW) of less than $1,000 \mathrm{~g}$ who were hospitalized in the NICU at the Institute for Child and Youth Health Care of Vojvodina during the 1-y period (01 January 2013 to 31 December 2013). Newborns who died during the first $72 \mathrm{~h}$ of life and those with congenital malformations of urinary system $(n=8)$ were excluded from the study. The total number of newborns who meet the criteria for entry into the study was the 22 .

AKI was diagnosed using modificated AKIN staging system (12). Increase of SCr levels of $\geq 26.5 \mu \mathrm{mol} / \mathrm{l}(\geq 0.3 \mathrm{mg} / \mathrm{dl})$ compared to the basal values on the third day of life, or if basal value was not determined-as an increase in the SCr levels of $\geq 26.5 \mu \mathrm{mol} / \mathrm{l}(\geq 0.3 \mathrm{mg} /$ dl) within $48 \mathrm{~h}$. Also, urine output criteria were not used for definition of AKI, as premature infants often have nonoliguric renal injury due to immature tubular development. The control group consisted of ELBW neonates who did not have AKI.

Basal SCr levels were determined in all neonates enrolled in the study in first $3 \mathrm{~d}$ of life. From fourth to seventh day of life, the $\mathrm{SCr}$ levels were measured in 24-48 h intervals. Beyond this period, if any clinical deterioration or signs of development of complications (sepsis, intracranial hemorrhage, patent ductus arteriosus, necrotizing enterocolitis) were present, SCr levels were monitored, at least, in the next $48 \mathrm{~h}$.

SCr levels were measured using modified kinetic Jaffe reaction method. Daily urine output was determined by collecting urine by urinary catheter or urine collecting bag (colector) or by measuring the weight of the diapers. Oliguria was defined as a urine output $<1 \mathrm{ml} / \mathrm{kg} / \mathrm{h}$.

Detection and quantification of urinary KIM-1 was done on the third day of life, using commertialy avalaible KIM-1 rapid test (Human KIM-1/Rena-strip Kit, Ijamsville, MD). Subsequently, measurements were repeated only in subjects who were diagnosed with $\mathrm{AKI}$, at different values of SCr.

The BioAssay Works H-rena-strip test is a 15-min test for the qualitative detection of KIM-1 in human urine. Seventy-five microliters of urine were diluted in clean test tube with the same volume of sample diluent buffer, mix, and then $100 \mu$ l of solution was put in the Rrena-cassette sample well. The presence of detectable levels of the KIM-1 in the test sample results in the formation of red test-lines within $15 \mathrm{~min}$. Different intensity and number of red test-lines represent different concentration of KIM-1, from $125 \mathrm{pg} / \mathrm{ml}$ to $60,000 \mathrm{pg} /$ $\mathrm{ml}$. Semiquantitative interpretation of the results was done refering to the color chart provided by the manufacturer.

After the death, histopathological evaluations of eight patiens' kidneys were performed. A semiquantative scale (histologic injury score) was used to evaluate kidney injury. The following histological features were used to assess the damage:

1. Tubular changes: degree of cellular damage was determinated separately for proximal and distal tubules. Changes were scored as 0 , no damage; 1 , minimal damage; 2 , moderately severe damage; 3 , severe damage.

2. Interstitial changes: presence of interstitial edema was scored as 0 , no edema; 1 , light edema; 2 , moderately prominent edema; 3, marked edema.

\section{Data Collection}

For all patients included in the study, following data were gathered and analyzed from medical records: GA; BW; gender; AS in the first and fifth minute; daily urine output; daily serum sodium levels during the first $4 \mathrm{~d}$ of life; usage of nephrotoxic drugs (for a group of neonates with AKI before diagnosing AKI) (aminoglycosides, ceftazidime, vancomycin, colomycine, nonsteroidal anti-inflammatory drugs, use of inotropes and furosemide, comorbidity (for a group of patients with AKI before diagnosing AKI) (sepsis, intracranial hemorrhage, patent ductus arteriosus, necrotizing enterocolitis). We also analyzed the outcome (death/survival).

Parental informed consent was obtained, and the study was approved by the Institutional Review Board at Institute for Child and Youth Health Care of Vojvodina.

\section{Statistics}

Descriptive data are presented as absolute numbers, frequencies, percentages, mean values \pm two $\mathrm{SD}$, minimum and maximum, the most common (mode) or central (median) values. Data are presented in text and tables. Inferential statistics included parametric and nonparametric statistical tests. To test the statistical differences between the groups, Student $t$-test was used for numerical variables and Fisher's exact probability test was used to test nominal variables. To test the correlation, linear regression (for numeric variables) and Spearman rank correlation (for ordinal variables) were used.

\section{STATEMENT OF FINANCIAL SUPPORT}

The study is performed as part of the project ON175079-3: "The role of biomarkers in early detection of renal impairment and monitoring of therapeutic response in children" supported by Ministry of Education, Science and Technological Development of Republic of Serbia.

Disclosures: The authors have no conflict of interest to disclose.

\section{REFERENCES}

1. Cataldi L, Leone R, Moretti U, et al. Potential risk factors for the development of acute renal failure in preterm newborn infants: a case-control study. Arch Dis Child Fetal Neonatal Ed 2005;90:F514-9.

2. Kungwani AR, Nimbalkar AS, Sethi AR, Patel DV, Nimbalkar SM. 1324 clinical profile and outcome of newborns with acute kidney injury in a level 3 neonatal unit in Western India. Arch Dis Child 2012;97:A377.

3. Agras PI, Tarcan A, Baskin E, Cengiz N, Gürakan B, Saatci U. Acute renal failure in the neonatal period. Ren Fail 2004;26:305-9.

4. Akcan-Arikan A, Zappitelli M, Loftis LL, Washburn KK, Jefferson LS, Goldstein SL. Modified RIFLE criteria in critically ill children with acute kidney injury. Kidney Int 2007;71:1028-35.

5. Elapavaluru S, Kellum JA. Why do patients die of acute kidney injury? Acta Clin Belg 2007;Suppl 2:326-31.

6. Askenazi DJ, Montesanti A, Hunley H, et al. Urine biomarkers predict acute kidney injury and mortality in very low birth weight infants. J Pediatr 2011;159:907-12.e1.

7. Andreoli SP. Acute renal failure in the newborn. Semin Perinatol 2004:28:112-23.

8. Abitbol CL, Bauer CR, Montané B, Chandar J, Duara S, Zilleruelo G. Longterm follow-up of extremely low birth weight infants with neonatal renal failure. Pediatr Nephrol 2003;18:887-93.

9. Askenazi DJ, Ambalavanan N, Goldstein SL. Acute kidney injury in critically ill newborns: what do we know? What do we need to learn? Pediatr Nephrol 2009;24:265-74.

10. Jetton JG, Askenazi DJ. Update on acute kidney injury in the neonate. Curr Opin Pediatr 2012;24:191-6.

11. Cuzzolin L, Fanos V, Pinna B, et al. Postnatal renal function in preterm newborns: a role of diseases, drugs and therapeutic interventions. Pediatr Nephrol 2006;21:931-8.

12. Koralkar R, Ambalavanan N, Levitan EB, McGwin G, Goldstein S, Askenazi D. Acute kidney injury reduces survival in very low birth weight infants. Pediatr Res 2011;69:354-8.

13. Vaidya VS, Ramirez V, Ichimura T, Bobadilla NA, Bonventre JV. Urinary kidney injury molecule-1: a sensitive quantitative biomarker for early detection of kidney tubular injury. Am J Physiol Renal Physiol 2006;290:F517-29.

14. Bonventre JV. Kidney injury molecule-1 (KIM-1): a urinary biomarker and much more. Nephrol Dial Transplant 2009;24:3265-8.

15. Han WK, Bailly V, Abichandani R, Thadhani R, Bonventre JV. Kidney Injury Molecule-1 (KIM-1): a novel biomarker for human renal proximal tubule injury. Kidney Int 2002;62:237-44.

16. Huang Y, Don-Wauchope AC. The clinical utility of kidney injury molecule 1 in the prediction, diagnosis and prognosis of acute kidney injury: a systematic review. Inflamm Allergy Drug Targets 2011;10:260-71.

17. Mehta RL, Kellum JA, Shah SV, et al.; Acute Kidney Injury Network. Acute Kidney Injury Network: report of an initiative to improve outcomes in acute kidney injury. Crit Care 2007;11:R31. 
18. Stapleton FB, Jones DP, Green RS. Acute renal failure in neonates: incidence, etiology and outcome. Pediatr Nephrol 1987;1:314-20.

19. Gupta BD, Sharma P, Bagla J, Parakh M, Soni JP. Renal failure in asphyxiated neonates. Indian Pediatr 2005;42:928-34.

20. Askenazi DJ, Griffin R, McGwin G, Carlo W, Ambalavanan N. Acute kidney injury is independently associated with mortality in very low birthweight infants: a matched case-control analysis. Pediatr Nephrol 2009;24:991-7.

21. Mathur NB, Agarwal HS, Maria A. Acute renal failure in neonatal sepsis. Indian J Pediatr 2006;73:499-502.

22. Aggarwal A, Kumar P, Chowdhary G, Majumdar S, Narang A. Evaluation of renal functions in asphyxiated newborns. J Trop Pediatr 2005;51:295-9.

23. Lunn AJ, Shaheen I, Watson AR. Acute renal insufficiency in the neonatal intensive care unit. Arch Dis Child Fetal Neonatal Ed 2006;91:F388.

24. Vachvanichsanong P, McNeil E, Dissaneevate S, Dissaneewate P, Chanvi$\tan \mathrm{P}$, Janjindamai W. Neonatal acute kidney injury in a tertiary center in a developing country. Nephrol Dial Transplant 2012;27:973-7.

25. Choker G, Gouyon JB. Diagnosis of acute renal failure in very preterm infants. Biol Neonate 2004;86:212-6.

26. Stojanović V, Barišić N, Milanović B, Doronjski A. Acute kidney injury in preterm infants admitted to a neonatal intensive care unit. Pediatr Nephrol 2014;29:2213-20.

27. Askenazi D. Evaluation and management of critically ill children with acute kidney injury. Curr Opin Pediatr 2011;23:201-7.

28. Coca SG, Yalavarthy R, Concato J, Parikh CR. Biomarkers for the diagnosis and risk stratification of acute kidney injury: a systematic review. Kidney Int 2008;73:1008-16.
29. Liborio AB, Castello Branco KMP, de Melo Bezerra T. Acute kidney injury in neonates: from urine output to new biomarkers. Biomed Res Int [online] 2014. http://dx.doi.org/10.1155/2014/601568.

30. Sarafidis K, Tsepkentzi E, Agakidou E, et al. Serum and urine acute kidney injury biomarkers in asphyxiated neonates. Pediatr Nephrol 2012;27: 1575-82.

31. Askenazi DJ, Koralkar R, Levitan EB, et al. Baseline values of candidate urine acute kidney injury biomarkers vary by gestational age in premature infants. Pediatr Res 2011;70:302-6.

32. van Timmeren MM, van den Heuvel MC, Bailly V, Bakker SJ, van Goor H, Stegeman CA. Tubular kidney injury molecule-1 (KIM-1) in human renal disease. J Pathol 2007;212:209-17.

33. Vaidya SV, Ford MG, Waikar SS, Wang Y, Clement BM, Ramirez V, et al. A rapid urine test for early detection of kidney injury. Kidney Int 2009;76:108-14.

34. Shao X, Tian L, Xu W, et al. Diagnostic value of urinary kidney injury molecule 1 for acute kidney injury: a meta-analysis. PLoS One 2014;9: e84131.

35. Liangos $\mathrm{O}$, Tighiouart $\mathrm{H}$, Perianayagam MC, et al. Comparative analysis of urinary biomarkers for early detection of acute kidney injury following cardiopulmonary bypass. Biomarkers 2009;14: $423-31$.

36. Stojanović VD, Vučković NM, Barišić NA, Srdić B, Doronjski AD, Peco Antić AE. Early biomarkers of renal injury and protective effect of erythropoietin on kidneys of asphyxiated newborn rats. Pediatr Res 2014;76:11-6. 